\title{
Antibiotic resistant Cutibacterium acnes among acne patients in Jordan: a cross sectional study
}

\author{
Eman Alkhawaja', Bayan Alkhawajaㄹ, Saleem Hammadi', Medhat Abdelmalek ${ }^{3}$, Naser Mahasneh ${ }^{4}$ and \\ Suzanne M. Abdelmalek ${ }^{1 *}$ (D)
}

\begin{abstract}
Background: Antibiotics have been used for decades against Cutibacterium acnes (previously known as Propionibacterium acnes; C. acnes). Alarmingly, antibiotic resistance to this bacterium has become a worldwide problem in recent years. No studies are available on the antibiotic susceptibility patterns of $C$. acnes among Jordanian acne patients and how that is influenced by antibiotic use.

This study aims to assess antibiotic resistance patterns of $C$. acnes clinical isolates and neighboring Gram-positive normal flora of the skin obtained from acne patients attending dermatology clinics in Amman -Jordan appraising the role of antibiotic consumption.

Methods: This is a cross-sectional study of acne patients presenting to selected dermatology outpatient clinics over a 6-month study period. Swabs obtained from inflamed lesions were cultured aerobically and anaerobically. Isolates were identified and screened for antibiotic susceptibility. In addition, all patients were asked to fill in a questionnaire that included questions about the history of antibiotic treatment.

Results: C. acnes was isolated from lesions of 100 patients out of 115 participants included in this study. $73 \%$ of the isolates were resistant to erythromycin and 59\% to clindamycin 37\% to doxycycline, $36 \%$ to tetracycline, $31 \%$ to trimethoprim / sulfamethoxazole, 15\% to levofloxacin, and 3\% to minocycline. Multi drug resistance (MDR) in C. acnes isolates as well as Staphylococcus aureus (S. aureus) and Staphylococcus epidermidis (S. epidermidis) with a similar pattern of resistance were detected from the same patient in most cases. A pattern of higher resistance towards variable antibiotic was observed in patients previously treated with antibiotics for acne management.

Conclusions: The findings of this study demonstrate the distribution of antibiotic resistance of $C$. acnes towards used antibiotics and emphasizes the influence of antibiotic consumption on development of antibiotic resistance. The similar pattern of resistance between skin bacteria tested in this study highlights the genetic transfer of resistance between skin commensals including S. aureus and S. epidermidis hence promoting its circulation in the community.
\end{abstract}

Keywords: Cutibacterium acnes, Acne, Antibiotic resistance patterns, Misuse of antibiotics, MDR

\footnotetext{
*Correspondence: sabdelmalek@uop.edu.jo

${ }^{1}$ Department of Pharmacology and Biomedical Sciences, Faculty of Pharmacy, Petra University, Amman, Jordan

Full list of author information is available at the end of the article
}

(c) The Author(s). 2020 Open Access This article is licensed under a Creative Commons Attribution 4.0 International License, which permits use, sharing, adaptation, distribution and reproduction in any medium or format, as long as you give appropriate credit to the original author(s) and the source, provide a link to the Creative Commons licence, and indicate if changes were made. The images or other third party material in this article are included in the article's Creative Commons licence, unless indicated otherwise in a credit line to the material. If material is not included in the article's Creative Commons licence and your intended use is not permitted by statutory regulation or exceeds the permitted use, you will need to obtain permission directly from the copyright holder. To view a copy of this licence, visit http://creativecommons.org/licenses/by/4.0/ The Creative Commons Public Domain Dedication waiver (http://creativecommons.org/publicdomain/zero/1.0/) applies to the data made available in this article, unless otherwise stated in a credit line to the data. 


\section{Background}

Acne is the most common dermatologic disorder in humans. It affects mostly adolescents (80\%) at the age of puberty when hormonal changes begin and impacts both genders similarly. Resolution of acne begins as patients reach their twenties, nevertheless; acne persists in 54\% of adult women and $40 \%$ of adult men [1, 2]. It follows the distribution of the sebaceous glands in the body hence it forms on the face, along the jaw line (which is a common location in adults), neck, chest, and back $[3,4]$. The disease manifests as white comedons, black heads, moderate acne, or inflamed acne. The pathogenesis of acne is complicated; it develops as a result of hormonal imbalance leading to overproduction of sebum accompanied by follicular duct hyper-keratinisation. The accumulation of dead skin and fatty acids pave the way for Cutibacterium acnes (C. acnes) follicular enhanced colonization and the subsequent release of inflammatory mediators $[5,6]$. C. acnes, commonly known as the cause of acne vulgaris, is typically an aerotolerant anaerobic, Gram-positive bacillus that colonizes the pilosebaceous follicle, derives energy from hydrolyzing triglycerides of the sebum, and secretes fatty acid byproducts, mainly propionic acid. These byproducts can irritate the follicular wall and induce inflammation through neutrophilic chemotaxis [7]. C. acnes promotes the abnormal proliferation and differentiation of keratinocytes as well as further sebum production. In addition, $C$. acnes activate the innate immune system through the overexpression of tolllike receptors, protease-activated receptors and matrix metalloproteinase by keratinocytes [8].

As a result of the complex pathogenicity of this condition, management is likewise diverse, including medications that control hormones, bacterial growth and the inflammatory response resulting from chemotactic factors produced by these bacteria. Control of bacterial growth is achieved by the use of antibiotics. Oral antibiotics are the standard treatment for moderate acne or for cases in which topical combinations were not tolerated or were ineffective [9]. Systemic erythromycin and different generations of tetracyclines are known to be effective in the management of inflammatory acne [10]. Vitamin A derivatives in the form of topical retinoids normalize the life cycle of follicles by preventing or reducing hyperkeritinization. Oral retinoid- (Isotretinoin) on the other hand, shrink the sebaceous glands, reduce sebum, cellular debris and metabolic byproducts from the surrounding skin tissue, which in turn reduces the amount of comedones produced $[5,11]$.

Given that both topical and oral antibiotics against $C$. acnes have been used for decades, $C$. acnes resistance to used antibiotics, such as erythromycin and clindamycin has been detected with high prevalence in Mediterranean countries mainly due to antibiotic abuse. For instance, researchers from Spain reported 91 and 92.4\% resistance respectively for both antibiotics. The rates of resistance in Greece were and $75.3 \%$ for both antibiotics and they were $59.5 \%$ in Italy [12]. The increasing number of $C$. acnes drug-resistant strains has provoked global concerns over the decreasing number of antibiotics that can be used to treat this common condition [13].

Antibiotic resistance in $C$. acnes can spread to other commensal bacteria colonizing the skin and the follicles such as $S$. epidermidis through horizontal transfer of resistance genes $[14,15]$. Therefore, careless administration of antibiotics would not only result in emerging antibiotic resistant $C$. acnes but also the spread of resistance to other bacterial species [14].

So far, there is no available data regarding antibiotic resistance of $C$. acnes among Jordanians. This study aims to determine the prevalence of antibiotic resistance in $C$. acnes isolates from acne patients attending private and public dermatology clinics in Amman -Jordan.

\section{Methods \\ Patients}

115 acne outpatients attending private and public dermatology clinics in Amman, Jordan were included in this study throughout a 6-month study period. Participants were asked to sign a consent form that included the methodology of the research and requested their approval for obtaining a microbiological sample from their inflamed pustules.

\section{Sample collection}

A dermatologist or a trained nurse were asked to obtain the sample after decontaminating the skin and the infected areas with $70 \%$ alcohol. Sterile cotton swabs were used to obtain the samples from a lesion. The lesion was opened aseptically in the clinic and swabbed. Swabs were aseptically placed in a Thioglycolate media tube (HI media-India). Another swab was obtained from the adjacent skin to the lesion. Swabs were sent to the lab for further analysis.

\section{Microbiological isolation and identification}

Thioglycolate tubes containing inoculated sample swabs were incubated at $37^{\circ} \mathrm{C}$ for 7 days and then sub cultured on blood agar aerobically and anaerobically using OXOID Anaerobic jar containing Anaerogen kit (OxoidGermany) for 7 days at $37^{\circ} \mathrm{C}$. Identification of bacterial isolates was performed according to colonial morphology, gram stain and biochemical reactions using RapID ANA п system (REMEL-USA).

\section{Antibiotic susceptibility testing}

Antibiotic susceptibility of isolates was determined by the disc diffusion assay according to the Clinical and 
Laboratory Standards Institute (CLSI) guidelines with some modifications. Briefly, five colonies were picked from blood agar culture and emulsified in $5 \mathrm{ml}$ of Mueller Hinton Broth MHB (Himedia- India) [12, 16]. A sterile swab was soaked in the suspension and seeded on the surface of Mueller Hinton Agar (MHA). Antibiotic discs $30 \mu \mathrm{g}$ tetracycline $(\mathrm{TE}), 30 \mu \mathrm{g}$ minocycline $(\mathrm{MH}), 30 \mu \mathrm{g}$ doxycycline (DO), $15 \mu \mathrm{g}$ erythromycin (E), $2 \mu \mathrm{g}$ clindamycin (DA), $1.25 / 23.75 \mu \mathrm{g}$ trimethoprim/sulfamethoxazole (SXT), $5 \mu \mathrm{g}$ Levofloxacin (LEV) (Oxoid (Germany) were then applied on the surface of the agar. Plates were incubated for 7 days in anaerobic conditions for $C$. acnes and overnight for other isolates. At the end of the incubation period, zones of inhibition were measured in millimeters. Experiments were performed in triplicates. The results were interpreted as resistant or sensitive according to the zone diameter interpretive chart (as per NCCLS January 2015) presented by Abdelfattah, Darwish, and Zandi et al. $[12,17,18]$

\section{History of antibiotic consumption}

A questionnaire was distributed to the dermatologist or the trained nurse to collect data from patients. The questionnaire was divided into three sections: first section demography which included age, gender and type of clinic whether private or public Second section: acne classification whether mild: few to several pustules and papules or moderate: several to many pustules with few nodules or severe: several to many papules and pustules with many nodules. The third section was history of antibiotics use: which asked if the patient had been treated with antibiotics during the prior year or ever, whether oral, topical, or combination antibiotics were used, duration of treatment, and the type of antibiotic used.

\section{Statistical analysis}

The data collected were coded and entered into SPSS 17.0 software. Descriptive analysis (frequencies, mean and standard deviation) as well as statistical significance testing was performed using chi-square and t-test tests. The statistical significance was defined as a $P$ value $\leq 0.05$.

\section{Results}

\section{Patients demographics}

Samples were obtained from 115 patients visiting public and private dermatology clinics. Eighty-seven patients were females while twenty-eight were males with a female to male ration of $2: 1$. The mean age of patients was $20.5 \pm 4$ years and the age of $63 \%$ of patients was equal to or greater than 20 years.

The patients experienced various degrees of severity of acne with moderate pustular acne being the predominant amongst them. Percentage of female patients with severe acne was higher than males.

C. acnes was isolated from 100 patients; 76 females with mean age $20 \pm 3.5$ years and 24 males with mean age $19 \pm 4.9$ years. Twenty-seven $(27 \%)$ patients of those had mild pustular acne, 57 (57\%) had moderate pustular acne and $16(16 \%)$ patients had severe pustular acne. The relationship between gender and acne severity was demonstrated in Fig. 1. The severity of acne diminished with age as patients $\geq 20$ years exhibited less severe acne (Fig. 2).

\section{Microorganisms isolated from acne patients}

One hundred and fifteen patients were enrolled in this study. C. acnes strains were isolated from 100 (87\%) patients; Staphylococcus epidermidis strains were also isolated together with C. acnes from the skin of 42 (36.5\%)

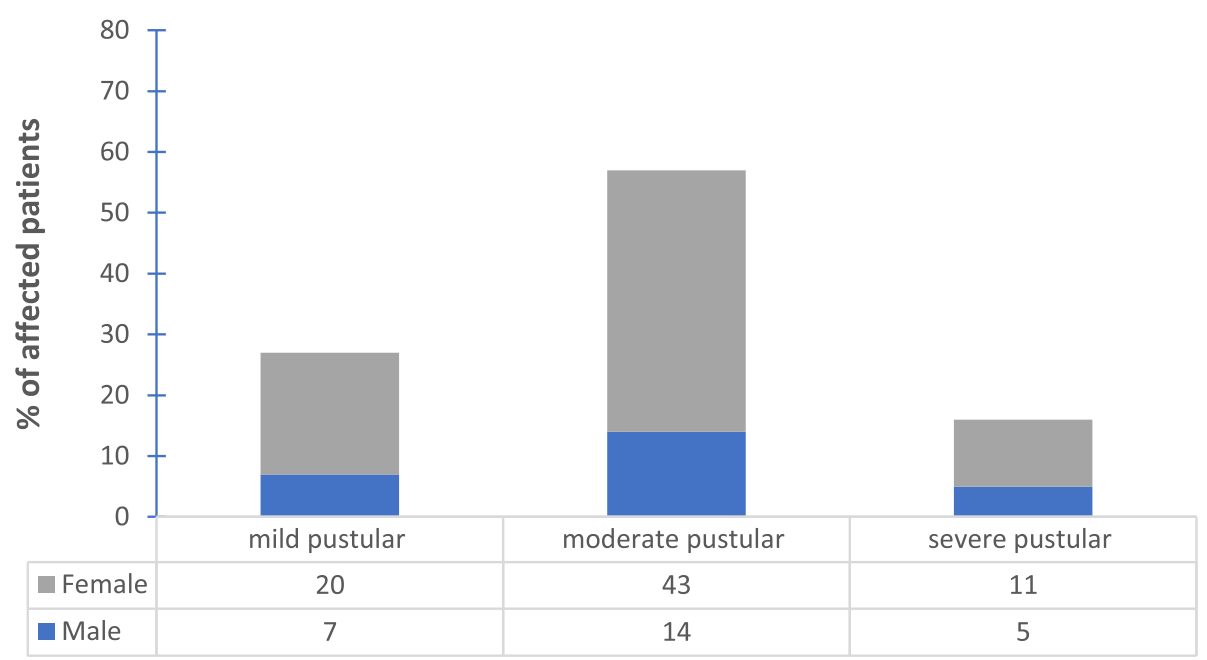

Fig. 1 The relation between gender and severity of acne 


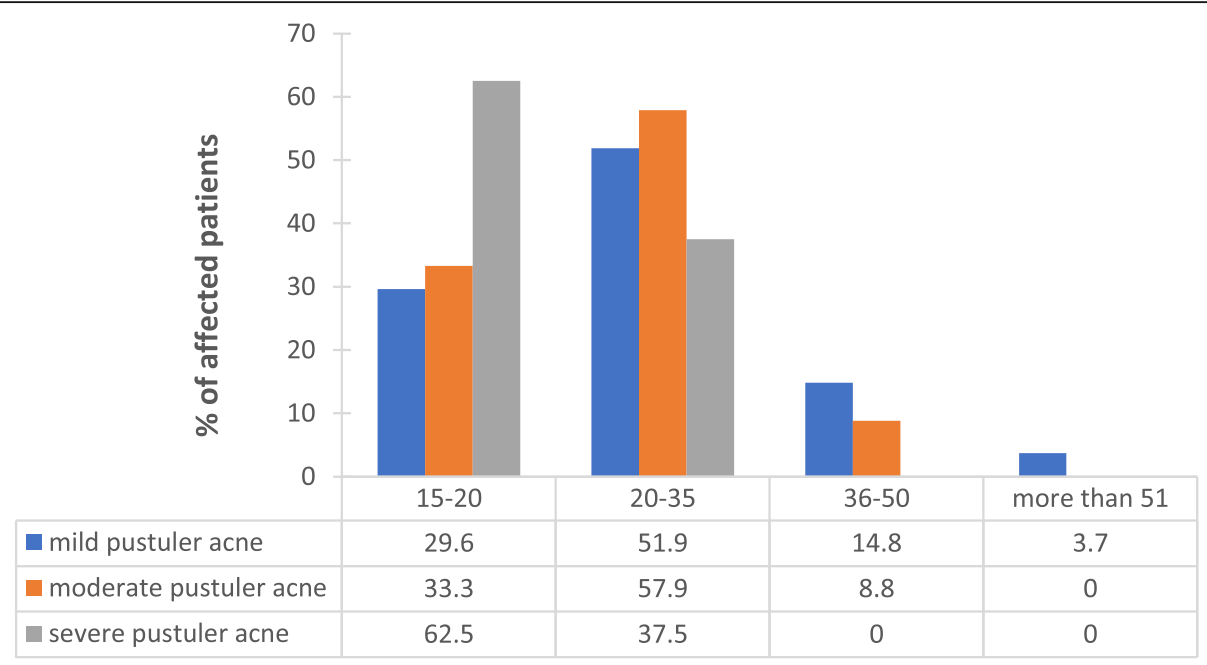

Fig. 2 The relation between age of patient and severity of acne condition

patients, while Staphylococcus aureus stains were isolated from 49 (42.6) patients. The three stains were isolated from 7 (6.1\%) patients (Table 1).

\section{Antimicrobial susceptibility of $C$. acnes isolated from acne patients}

C. acnes was isolated from lesions of $87 \%$ of acne patients. $100 \%$ of the isolates showed reduced susceptibility to one or more than one antibiotic.

Clinical isolate strains of $C$. acnes showed variable resistance to the used antibiotics. It ranged from resistance to one antibiotic to multidrug resistance that involved different antibiotics having different mode of actions (Fig. 3). Erythromycin and clindamycin exhibited the highest resistance percentages among tested antibiotics (Table 2).

The number of patients carrying antibiotic resistant $C$. acnes and attending private clinics exceeded those attending public clinics, however this difference was not significant and conclusions cannot be drawn from it due to the small sample size and potential confounders (Fig. 4).

\section{Antibiotics history}

Regarding previous use of antibiotic therapy for acne treatment, patients were divided into two groups: patients who did not receive prior treatment for acne 39

Table 1 Distribution of bacterial isolates from patients

\begin{tabular}{llll}
\hline $\begin{array}{l}\text { Number of } \\
\text { samples }\end{array}$ & $\begin{array}{l}\text { Acne lesion } \\
\text { C. acnes }\end{array}$ & skin \\
\cline { 2 - 4 } & + & - & S. aureus \\
\hline 42 & + & + & S. epidermidis \\
49 & + & + & - \\
7 & + & - & + \\
2 & - & - & - \\
15 & & & - \\
\hline
\end{tabular}

(39\%) and patients who had received prior antibiotic therapy 61 (61\%). The with mean treatment duration for patients who had received prior therapy was $6 \pm 2$ weeks. Thirty-one of those patients $(50.8 \%)$ had received topical antibiotics (clindamycin (29.1\%) and erythromycin (70.9\%)), 17 patients $(27.9 \%)$ were treated with systemic antibiotics (doxycycline) and $13(21.3 \%)$ patients were treated with both oral and topical antibiotics simultaneously (Fig. 5).

The distribution of resistance in C. acnes isolates was mainly towards erythromycin, doxycycline and clindamycin which are the most commonly administrated antibiotics for acne management between patients (Table 2). No significant difference was found between the two groups in their resistance profile towards the tested antibiotics yet a higher number of resistant isolates with variable antibiotic resistance was observed in the previously treated group.

Antimicrobial susceptibility of S. aureus and S. epidermidis isolated from acne patients

S. epidermidis and S. aureus were isolated from the skin of $85.5 \%$ of patients. The antimicrobial susceptibility patterns from the total sample (91 patients) are shown in (Table 3).

Isolates that showed cross-resistance with $C$. acnes were isolated (data not shown). Four isolates showed cross-resistance towards erythromycin between the three studied stains while three isolates showed cross resistance between strains towards clindamycin.

Resistance to antibiotics varied between isolated strains. It ranged from resistance to one antibiotic to resistance to several antibiotics having different modes of action (Fig. 3). Of the 91 isolates of both S. epidermidis 


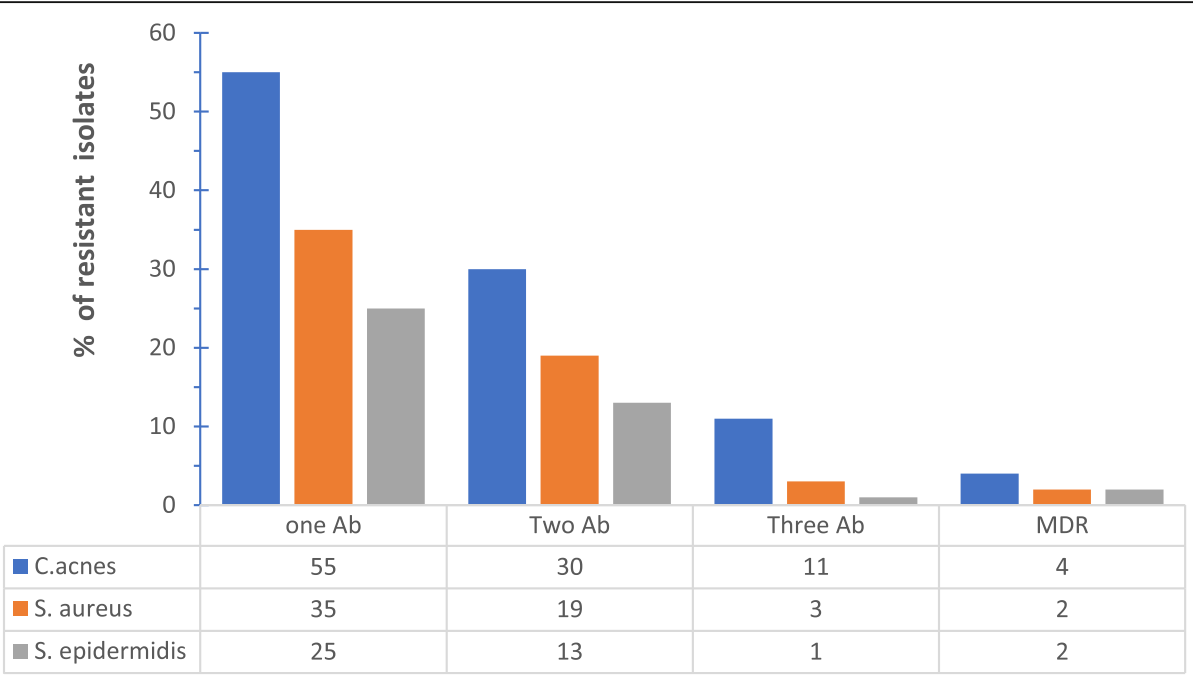

Fig. 3 Percentage of resistant isolates to antibiotics. Where Ab: antibiotic, MDR: multi-drug resistance

and $S$. aureus, antibiotic resistance was more prevalent in isolates from private clinic patients (Fig. 6).

\section{Discussion}

This study aimed to assess the prevalence of antibiotic resistant $C$. acnes among a sample of Jordanian patients. While other studies showed that severe acne is more common in the second and third decade and may persist into their sixth decade $[4,5,19], 62.5 \%$ of severe pustular acne was seen between the age of 15-20 in our cohort. This might be related to hormonal changes as well as stresses that this age group undergoes due to high school/college entry exams $[20,21]$, a well-known stressful period for this age group in Jordan. Moreover, the decreasing severity of acne with age is attributed to the reduction in sebaceous gland activity compared to puberty [22]. Incidence of acne in middle-aged and older adults (above 35 yrs.) was minimal with the highest incidence being at age range between 15 and 35 yrs. (Fig. 2). This pattern of acne distribution was also demonstrated by others $[12,16]$.
C. acnes was isolated from 100 samples with a recovery rate of $86.9 \%$. Staphylococcus species, including $S$. epidermidis and $S$. aureus were isolated from the skin of patients harboring $C$. acnes (Table 1). The presence of Staphylococcus aureus as a transient normal flora of the skin is well documented with high carrier rates in the population $[15,23]$.

S. epidermidis has been reported to aggravate inflammatory acne and an imbalance of skin commensals in favor of S. epidermidis could aggravate inflammation of the sebaceous unit as a consequence [16]. Claudel et al have recently reported a potential beneficial role of $S$. epidermidis arguing that acne results from a disturbed equilibrium between $C$. acnes and S. epidermidis in pilosebaceous units of acne [24]. S. epidermidis controls the proliferation of $C$. acnes via the release of succinic acid, a fatty acid fermentation product, which inhibits surface Toll-like receptors of keratinocytes and tumor necrosis factor and suppresses C. acnes-induced IL-6 [24, 25].

Although C. acnes are known to be very sensitive to a wide range of antibiotic classes, including clindamycin, erythromycin, and cyclines, the percentage of acne

Table 2 Percentage of $C$. acnes isolates resistant to different antibiotics

\begin{tabular}{llll}
\hline Antibiotic & Concentration $(\boldsymbol{\mu g})$ & $\begin{array}{l}\text { Sensitive }^{\mathbf{a}} \\
\mathbf{n}(\%)\end{array}$ & $\begin{array}{l}\mathbf{R e s i s t a n t}^{\mathbf{a}} \\
\mathbf{n}(\%)\end{array}$ \\
\hline Doxycycline & 30 & $(63) 63 \%$ & $(37) 37 \%$ \\
Tetracycline & 30 & $(64) 64 \%$ & $(36) 36 \%$ \\
Erythromycin & 15 & $(27) 27 \%$ & $(73) 73 \%$ \\
Clindamycin & 2 & $(41) 41 \%$ & $(59) 59 \%$ \\
Trimethoprim/sulfamethoxazole & $12.5 / 23.75$ & $(69) 69 \%$ & $(31) 31 \%$ \\
Levofloxacin & 5 & $(85) 85 \%$ & $(15) 15 \%$ \\
Minocycline & 30 & $(97) 97 \%$ & $(3) 3 \%$ \\
\hline
\end{tabular}

${ }^{\mathrm{a}}$ Determined according to breakpoints ranges $[12,17,18]$. 


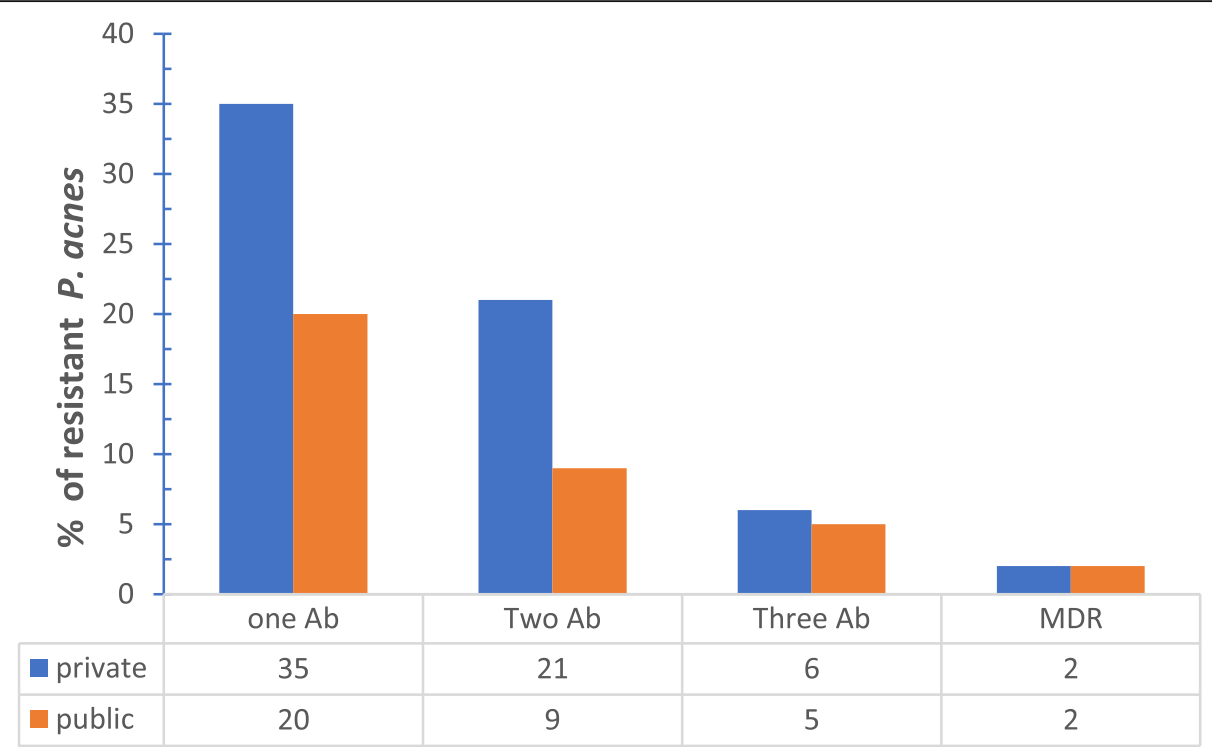

Fig. 4 Distribution of single and multiple antibiotic resistant $P$. acnes strains between private and public clinics. Where Ab: antibiotic, MDR: multi-drug resistance

patients carrying C. acnes strains resistant to these antibiotics is increasing worldwide and differ from one region to another [26-28]. In this study all 100 patients harbored $C$. acnes with varying degrees of resistance to variable antibiotics. Resistance to erythromycin and clindamycin was the highest while isolates were relatively susceptible to tetracycline (Table 2). These results are consistent with the overall resistance pattern throughout the world $[12,28,29]$.
Antibiotic resistance patterns varied between isolates. Some C. acnes isolates demonstrated resistance to one antibiotic, others to two different antibiotics and yet a third group demonstrated multi-drug resistance towards three different antibiotics (Fig. 3). Multi-drug resistant $C$. acnes was also reported recently by Zhang et al who isolated strains that were simultaneously resistant to clindamycin, erythromycin and moxifloxacin [6].

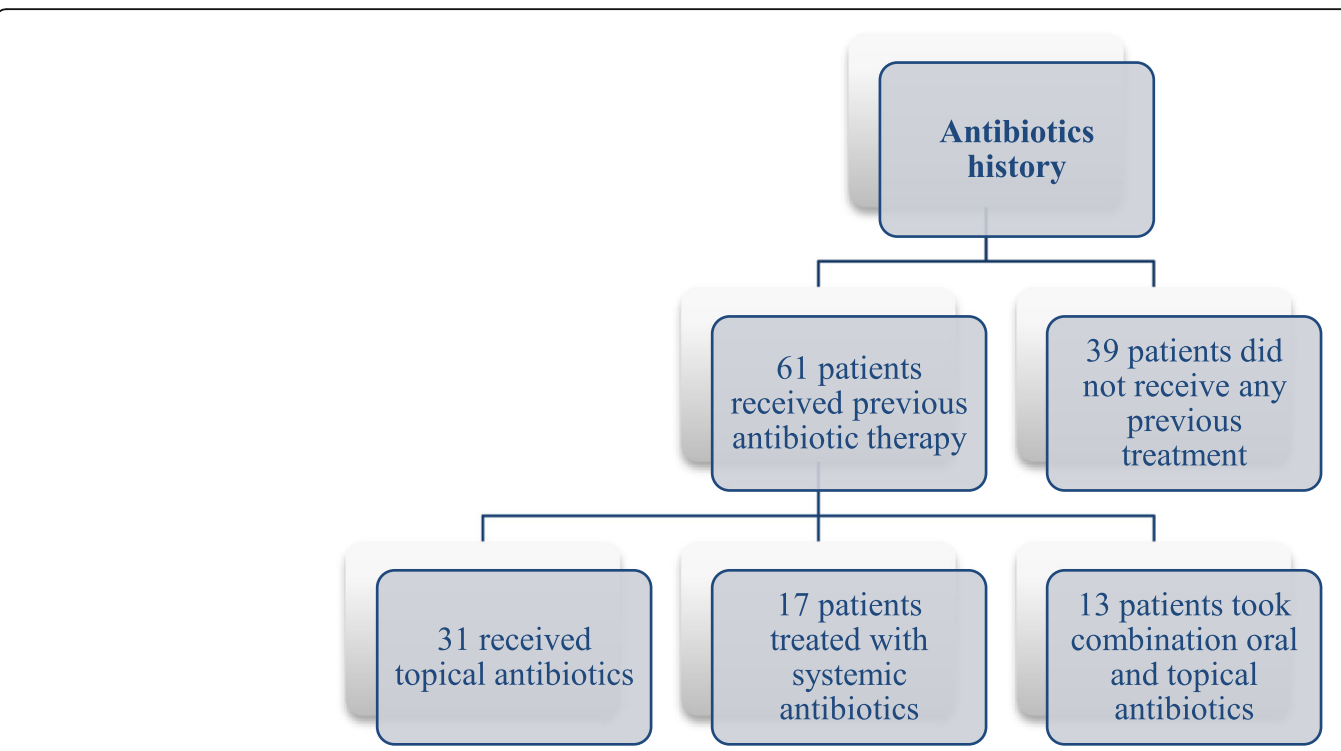

Fig. 5 Antibiotic administration history amongst the patients 
Table 3 Antibiotic susceptibilities of isolated S. aureus and S. epidermidis

\begin{tabular}{|c|c|c|c|c|c|c|}
\hline \multirow[t]{2}{*}{ Antibiotic } & \multicolumn{2}{|c|}{ Sensitive (\%) ${ }^{\mathrm{a}}$} & \multicolumn{2}{|c|}{ Resistant (\%) ${ }^{\mathrm{a}}$} & \multicolumn{2}{|c|}{ Cross-resistance with C. acnes (\%) } \\
\hline & S. aureus & S. epidermidis & S. aureus & S. epidermidis & S. aureus & S. epidermidis \\
\hline Doxycyclin & $26(53 \%)$ & $18(43 \%)$ & $23(47 \%)$ & $24(57 \%)$ & $6(12 \%)$ & $4(10 \%)$ \\
\hline Tetracyclin & $33(67 \%)$ & $22(52 \%)$ & $16(33 \%)$ & $20(48 \%)$ & $5(10 \%)$ & $3(7 \%)$ \\
\hline Erythromycin & $11(22 \%)$ & $4(10 \%)$ & $38(78 \%)$ & $38(90 \%)$ & $11(22 \%)$ & $10(23 \%)$ \\
\hline clindamycin & $18(37 \%)$ & $7(17 \%)$ & $31(63 \%)$ & $35(83 \%)$ & $9(18 \%)$ & $8(19 \%)$ \\
\hline Trimethoprim / sulfamethoxazole & $23(47 \%)$ & $11(26 \%)$ & $26(53 \%)$ & $31(74 \%)$ & $5(10 \%)$ & $5(12 \%)$ \\
\hline Levofloxacin & $44(90 \%)$ & $30(71 \%)$ & $5(10 \%)$ & $12(29 \%)$ & - & - \\
\hline Minocycline & $45(92 \%)$ & $32(76 \%)$ & $4(8 \%)$ & $10(24 \%)$ & - & - \\
\hline
\end{tabular}

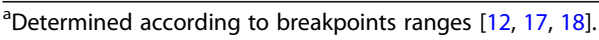

Similarly, this pattern was also found in the staphylococcal strains isolated from the skin of patients and not from the acne lesions themselves (Fig. 3). Antibiotic resistance patterns between isolates from the same patient have shown similarity (Table 3). Multiple resistances often included resistance towards erythromycin and clindamycin. Cross-resistance between erythromycin and clindamycin is thought to be due to the presence of the erm $(\mathrm{X})$ resistance gene that confers resistance to Macrolide, Lincosamide and Streptogramin B (MLS) group of antibiotics [30]. This explains the similarity in antibiotic resistance patterns between isolates from the same patient suggesting gene- transfer between species. S. epidermidis ability to transfer genes via plasmids to other more pathogenic staphylococcal species has been documented [3].

Most $C$. acnes isolates were resistant to the major five antibiotics (erythromycin, clindamycin, doxycycline, trimethoprim / sulfamethoxazole, and tetracycline). However, 97 and $85 \%$ of isolates were susceptible to minocycline and levofloxacin respectively (Table 2). Minocycline and levofloxacin are not used frequently in Jordan due to cost. Minocycline's side effect profile also includes irreversible slate-grey hyper pigmentation of the skin and the development of lupus-like syndrome which both make it a less commonly used agent for the treatment of acne [31]. The high susceptibility rates towards minocycline and levofloxacin accompanied with high resistance rates towards other antibiotics highlights the excessive use/abuse of the latter antibiotics. The high resistance rates towards erythromycin, clindamycin and doxycycline are similar to previously published reports and mirror the antibiotic-use history for the studied patients (Fig. 5). The presence of such resistant strains could explain the clinical unresponsiveness of these patients as well as the high relapse frequency.

It has been previously demonstrated that the use of topical antibiotics in acne results in antibiotic resistance if used for long periods (more than 8 weeks) [5, 32, 33]. Similarly, $21 \%$ of $C$. acnes resistance to clindamycin and erythromycin in our study was detected in isolates from patients who used topical antibiotics (results not shown).

In contrast to the majority of patients visiting the public dermatology clinics, patients attending private clinic were previously treated with antibiotics for acne management. This is reflected in the distribution of resistant C. acnes isolate. Private clinic patients showed a higher percentage of resistant $C$. acnes than public clinic patients (Fig. 4). This pattern of distribution of resistant strains was not restricted to $C$. acnes but extended to $S$.

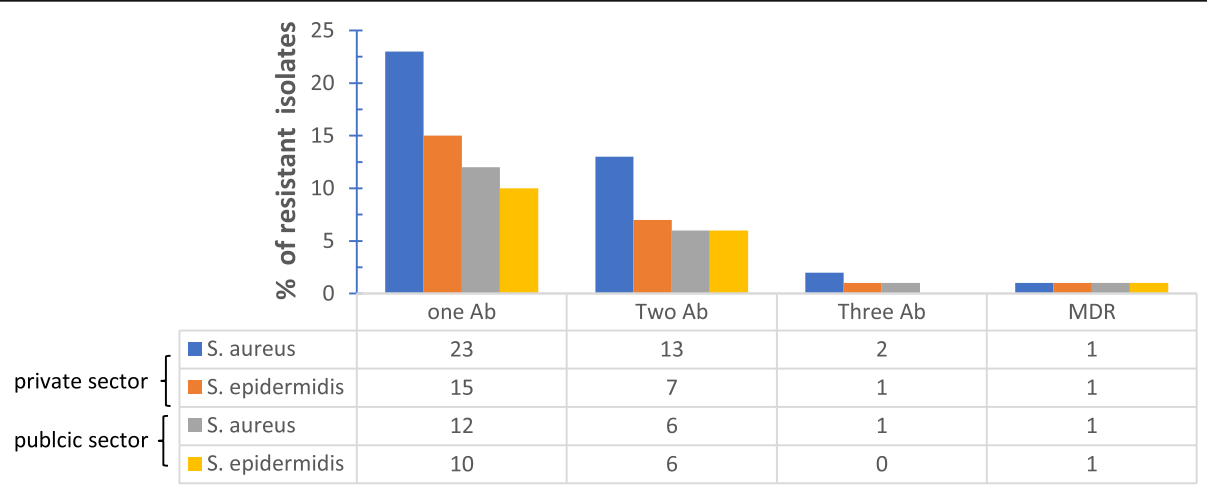

Fig. 6 Percentage of S. aureus and S. epidermidis resistance to single and multiple antibiotics in private and public sectors. Where Ab: antibiotic, MDR: multi-drug resistance 
epidermidis and S. aureus (Fig. 6). Thereby, we could correlate prior consumption of antibiotics with higher resistance patterns observed amongst private sector patients. Significant correlation was found between previous antibiotic administration with the prevalence of $\mathrm{C}$. acnes resistant strains [34].

Thirty-nine percent of the patients in this study did not receive prior antibiotic treatment, while $(61 \%)$ of patients received prior antibiotic therapy with mean treatment duration of $6 \pm 2$ weeks (Fig. 5). All non-previously treated patients carried antibiotic resistant strains, similarly to previously treated ones, indicating that antibiotic resistant strains are circulating and are emerging not only from acne treatment but also as a result of antibiotic use in treatment of other diseases.

The presence of antibiotic resistance in the normal flora of outpatients highlights the possibility of community spread of antibiotic resistant strains through horizontal transfer. In accordance with previous studies, the relationship between resistance and history of antibiotic use remains debated and resistance to antibiotics could be mainly acquired due to contact-mediated spread of resistant $C$. acnes $[6,35]$.

Comparing the resistance status of $C$. acnes in Jordan to that in other countries; resistance in Jordan was above average for erythromycin, clindamycin, and doxycycline, and almost similar in the case of minocycline. Such results indicate that the frequent use of certain antibiotics has led to the emergence of resistant strains in comparison to minocycline that is not used as much. Outside of acne, erythromycin and clindamycin are commonly used antibiotics. Erythromycin is used in the treatment of upper respiratory tract infections caused by M. pneumoniae, Legionella species, beta-hemolytic streptococcus, and Streptococcus pneumoniae. Clindamycin is bactericidal to most non enterococcal gram-positive aerobic bacteria and many anaerobic microorganisms and is considered an excellent alternative to beta-lactam antibiotics for treatment of many staphylococcal infection [36]. The co-existence of $C$.acnes resistant strains with a similar resistance pattern indicates the likelihood of horizontal transfer of antibiotic resistance genes across different bacterial species as noted earlier.

In conclusion, our study demonstrates that the prevalence of $C$. acnes antibiotic-resistance in Jordan is above average compared to other countries. We showed higher resistance of $C$. acnes isolates to erythromycin and clindamycin and intermediate resistance to tetracycline, doxycycline, and trimethoprim/sulfamethoxazole. Furthermore, we demonstrated the presence of multi-drug resistant C.acnes strains in outpatients in Jordan. Levofloxacin and minocycline demonstrated the best in-vitro activity against the $C$. acnes isolates. Finally, S. aureus and S. epidermidis was also isolated from acne patients and demonstrated a similar antibiotic resistance pattern to $C$. acnes.

\section{Supplementary information}

Supplementary information accompanies this paper at https://doi.org/10. 1186/s12895-020-00108-9.

\section{Additional file 1.}

\section{Abbreviations}

Ab: Antibiotic; DA: Clindamycin; CLSI: Clinical and Laboratory Standards Institute; C. acnes: Cutibacterium acnes; DO: Doxycycline; E: Erythromycin; LEV: Levofloxacin; MLS: Macrolide, Lincosamide and Streptogramin B; MH: Minocycline; MDR: Multi drug resistance; S. aureus: Staphylococcus aureus; S. epidermidis: Staphylococcus epidermidis; TE: Tetracycline;

SXT: Trimethoprim/sulfamethoxazole

\section{Acknowledgments}

The authors would like to express their appreciation and gratitude to Dr. Yousef Badran for copy editing the manuscript and extend their appreciation to all patients who agreed to participate in this study and to the Deanship of Scientific research at the University of Petra for facilitating all stages of this research.

\section{Declarations}

The study was approved by the Institutional Ethical Review Board (IRB) at the University of Petra (10H-11-2018). Informed written consent was provided by all participants.

\section{Authors' contributions}

SA and SH Conception and design of the work, interpretation of data; EA Data collection and analysis, MA and NM Critical revision of the article. BA have drafted the work. All authors have contributed to the final approval of the version to be published.

\section{Funding}

No funding.

\section{Availability of data and materials}

The datasets used and/or analyzed during the current study are available from the corresponding author on reasonable request.

\section{Consent for publication \\ Not applicable.}

\section{Competing interests}

The authors declare that they have no competing interests.

\section{Author details}

${ }^{1}$ Department of Pharmacology and Biomedical Sciences, Faculty of Pharmacy, Petra University, Amman, Jordan. ²Department of Pharmaceutical Medicinal Chemistry \& Pharmacognosy, Faculty of Pharmacy, Petra University, Amman, Jordan. ${ }^{3}$ Consultant Dermatologist, private clinic, Amman, Jordan. ${ }^{4}$ Department of Dermatology, Faculty of Medicine, Jordan University, Amman, Jordan.

Received: 5 June 2020 Accepted: 8 October 2020

Published online: 17 November 2020

References

1. Bhate K, Williams HC. Epidemiology of acne vulgaris. Br J Dermatol. 2013; 168:474-85. https://doi.org/10.1111/bjd.12149.

2. Ahmad N, Niazi K. Beliefs and perceptions about acne among undergraduate medical students. J Pakistan Assoc Dermatologists. 2007;17: 231-4.

3. Thiboutot D, Gollnick H, Bettoli V, Dréno B, Kang S, Leyden JJ, et al. New insights into the management of acne: an update from the global Alliance to improve outcomes in acne group. J Am Acad Dermatol. 2009;60(5 Suppl):S1-50.

4. Williams HC, Dellavalle RP, Garner S. Acne vulgaris. In: The Lancet. Elsevier; 2012. p. $361-72$. 
5. Nast A, Dréno B, Bettoli V, Degitz K, Erdmann R, Finlay AY, et al. European evidence-based (S3) guidelines for the treatment of acne. J Eur Acad Dermatol Venereol. 2012;26(SUPPL. 1):1-29.

6. Zhang N, Yuan R, Xin KZ, Lu Z, Ma Y. Antimicrobial Susceptibility, Biotypes and Phylotypes of Clinical Cutibacterium (Formerly Propionibacterium) acnes Strains Isolated from Acne Patients: An Observational Study. Dermatol Ther (Heidelb). 2019;9:735-46. https://doi. org/10.1007/s13555-019-00320-7.

7. Cogen AL, Nizet V, Gallo RL. Skin microbiota: a source of disease or defence? Br J Dermatol. 2008;158:442-55.

8. Beylot C, Auffret N, Poli F, Claudel JP, Leccia MT, Del Giudice P, et al. Propionibacterium acnes: an update on its role in the pathogenesis of acne. J Eur Acad Dermatol Venereol. 2014;28:271-8.

9. Titus S, Hodge J. Diagnosis and treatment of acne. Am Fam Physician. 2012; 86:734-40.

10. Pires MC, de Souza Sittart JA. Systemic antibiotics in acne. Expert Rev Dermatol. 2006:1:165-7.

11. Ghali F, Kang S, Leyden J, Shalita AR, Thiboutot DM. Changing the face of acne therapy. Cutis. 2009;83(2 Suppl):4-15.

12. Abdel Fattah NS a, Darwish YW. In vitro antibiotic susceptibility patterns of Propionibacterium acnes isolated from acne patients: an Egyptian university hospital-based study. J Eur Acad Dermatol Venereol. 2013;27:1546-51.

13. Walsh TR, Efthimiou J, Dréno B. Systematic review of antibiotic resistance in acne: an increasing topical and oral threat. Lancet Infect Dis. 2016;16:e23-33.

14. Jappe U. Pathological mechanisms of acne with special emphasis on Propionibacterium acnes and related therapy. Acta Derm Venereol. 2003;83: 241-8.

15. Fanelli M, Kupperman E, Lautenbach E, Edelstein PH, Margolis DJ. Antibiotics, acne, and Staphylococcus aureus colonization. Arch Dermatol. 2011;147:917-21.

16. Moon SH, Roh HS, Kim YH, Kim JE, Ko JY. Antibiotic resistance of microbial strains isolated from Korean acne patients; 2012. p. 833-7.

17. Zandi S, Vares B, Abdollahi H. Determination of microbial agents of acne vulgaris and Propionibacterium acnes antibiotic resistance in patients referred to dermatology clinics in Kerman, Iran; 2011.

18. National Committee for Clinical Laboratory Standards. Performance Standards for Antimicrobial Disk Susceptibility Tests; Approved StandardTwelfth Edition. M02-A12. 950 West Valley Road, Suite 2500 Wayne, PA 19087 USA; 2015. www.clsi.org.

19. Ramos-e-Silva M, Carneiro SC. Acne vulgaris: review and guidelines. Dermatol Nurs. 2009;21.

20. Chiu A, Chon SY, Kimball AB. The Response of Skin Disease to Stress. Arch Dermatol. 2003;139:897-900.

21. Yosipovitch G, Tang M, Dawn AG, Chen M, Goh CL, Chan YH, et al. Study of psychological stress, sebum production and acne vulgaris in adolescents. Acta Derm Venereol. 2007:87:135-9.

22. Zouboulis CC. Acne and sebaceous gland function. Clin Dermatol. 2004;22: 360-6.

23. Chambers HF. The changing epidemiology of Staphylococcus aureus? Emerg Infect Dis. 2001;7:178-82.

24. Claudel JP, Auffret N, Leccia MT, Poli F, Corvec S, Dréno B. Staphylococcus epidermidis: a potential new player in the physiopathology of acne? Dermatology. 2019;235:287-94.

25. Wang Y, Kuo S, Shu M, Yu J, Huang S, Dai A, et al. Staphylococcus epidermidis in the human skin microbiome mediates fermentation to inhibit the growth of Propionibacterium acnes: implications of probiotics in acne vulgaris. Appl Microbiol Biotechnol. 2014;98:411-24.

26. Coates P, Vyakrnam S, Eady EA, Jones CE, Cove JH, Cunliffe WJ. Clinical Investigations Prevalence of antibiotic-resistant propionibacteria on the skin of acne patients : 10-year surveillance data and snapshot distribution study. Br J Dermatol. 2002:840-8.

27. Magin PJ, Pond CD, Smith WT, Watson AB, Goode SM. A cross-sectional study of psychological morbidity in patients with acne, psoriasis and atopic dermatitis in specialist dermatology and general practices. J Eur Acad Dermatology Venereol. 2008;22:1435-44.

28. Ross Jl, Snelling AM, Carnegie E, Coates P, Cunliffe WJ, Bettoli V, et al. Antibiotic-resistant acne: lessons from Europe. Br J Dermatol. 2003;148:46778. https://doi.org/10.1046/j.1365-2133.2003.05067.x.

29. Nakase K, Nakaminami H, Takenaka Y, Hayashi N, Kawashima M, Noguchi N. Relationship between the severity of acne vulgaris and antimicrobia resistance of bacteria isolated from acne lesions in a hospital in Japan. J
Med Microbiol. 2014;63(PART 5):721-8. https://doi.org/10.1099/jmm.0. 067611-0.

30. Ross Jl, Carnegie E, Cove JH. Detection of transposon Tn5432-mediated macrolide-lincosamide-streptogramin B (MLSB) resistance in cutaneous propionibacteria from six European cities. - abstract - Europe PMC. J Antimicrob Chemother. 2002;49:165-8 https://europepmc.org/article/ med/11751782. Accessed 8 May 2020.

31. McManus $P$, Iheanacho I. Don't use minocycline as first line oral antibiotic in acne. BMJ. 2007:334:154

32. Humphrey S. Antibiotic resistance in acne treatment. Skin Therapy Lett. 2012;17:1-3.

33. Dreno B. Topical antibacterial therapy for acne vulgaris. Drugs. 2004;64: 2389-97.

34. Oprica C, Emtestam L, Lapins J, Borglund E, Nyberg F, Stenlund K, et al. Antibiotic-resistant Propionibacterium acnes on the skin of patients with moderate to severe acne in Stockholm. Anaerobe. 2004;10:155-64.

35. Schafer F, Fich F, Lam M, Gárate C, Wozniak A, Garcia P. Antimicrobial susceptibility and genetic characteristics of Propionibacterium acnes isolated from patients with acne. Int J Dermatol. 2013;52:418-25. https://doi. org/10.1111/j.1365-4632.2011.05371.x.

36. Smilack JD, Wilson WR, Cockerill FR. Tetracyclines, chloramphenicol, erythromycin, clindamycin, and metronidazole. Mayo Clin Proc. 1991;66: 1270-80.

\section{Publisher's Note}

Springer Nature remains neutral with regard to jurisdictional claims in published maps and institutional affiliations.
Ready to submit your research? Choose BMC and benefit from:

- fast, convenient online submission

- thorough peer review by experienced researchers in your field

- rapid publication on acceptance

- support for research data, including large and complex data types

- gold Open Access which fosters wider collaboration and increased citations

- maximum visibility for your research: over $100 \mathrm{M}$ website views per year

At BMC, research is always in progress.

Learn more biomedcentral.com/submissions 